DOI: https://doi.org/10.46296/yc.v6i10edespmar.0160

\title{
FACTORES QUE INFLUYEN EN LA REPITENCIA ESTUDIANTIL MEDIANTE EL ANÁLISIS DE CORRESPONDENCIA MÚLTIPLE
}

\section{FACTORS THAT INFLUENCE STUDENT REPEAT THROUGH MULTIPLE CORRESPONDENCE ANALYSIS}

\author{
Quimiz-Moreira Mauricio Alexander ${ }^{1}$; Márquez-Pérez Víctor Ernesto ${ }^{2}$ \\ ${ }^{1}$ Maestría en Estadística, Instituto de Posgrado, Universidad Técnica de Manabí, UTM. \\ Portoviejo, Ecuador. Correo: mauricio.quimiz@utm.edu.ec. \\ ${ }^{2}$ Departamento de Matemáticas y Estadística. Instituto de Ciencias Básicas, Universidad \\ Técnica de Manabí, UTM. Portoviejo, Ecuador. Correo: victor.marquez@utm.edu.ec.
}

\section{Resumen}

En la actualidad, la repitencia constituye un fenómeno que subsiste en las instituciones universitarias con diferentes magnitudes e implicaciones tanto en lo individual, lo colectivo y lo social. El presente artículo tiene como objetivo, analizar los factores que influyen en la repitencia mediante análisis de correspondencia múltiple, poniéndose de relieve que su ocurrencia está influenciada tanto por factores exógenos como endógenos, y que las universidades ecuatorianas deben priorizar la realización de investigaciones sobre este tema, que favorezcan el diseño e implementación de acciones para minimizar el efecto de la referida problemática. La investigación permite aseverar que este tema posee una elevada vigencia en el ámbito de la educación de tercer nivel en el Ecuador, que constata por las altas cifras de estudiantes que causan bajan cada o repiten en las diferentes carreras que se ofertan tanto en universidades públicas como privadas. Para el análisis de los datos se hizo uso del software estadístico SPSS, por pasos hacia atrás haciendo uso del criterio de Razón de Verosimilitud (RV) entre las variables dependiente (Repitencia Estudiantil) y las variables independientes (Socioeconómicas) y con un p-valor $<0.10$ dentro del test de Wald. Se obtuvo como conclusión Una vez realizada el análisis de la regresión logística binaria con un nivel de significación de $p<0.10$ se puede identificar que las variables TRABAJA y TIPO DE VIVIENDA cumple con el criterio mencionado y son variables de significancia en la problemática de deserción de estudiantes universitarios de la Facultad de Ciencias Informáticas de la Universidad Técnica de Manabí.

Palabras claves: Repitencia, Análisis de correspondencia múltiple, Universidad, variables sociodemográficas.

\begin{abstract}
Currently, repetition is a phenomenon that persists in university institutions with different magnitudes and implications both individually, collectively and socially. This article aims to analyze the factors that influence repetition through multiple correspondence analysis, highlighting that its occurrence is influenced by both exogenous and endogenous factors, and that Ecuadorian universities should prioritize research on this topic., that favor the design and implementation of actions to minimize the effect of the aforementioned problem. The research allows us to assert that this topic has a high validity in the field of third-level education in Ecuador, which is confirmed by the high numbers of students who drop each year or repeat in the different careers that are offered both in public universities and private. For the analysis of the data, the SPSS statistical software was used, by steps backwards using the Likelihood Ratio (RV) criterion between the dependent variables (Student Repetition) and the independent variables (Socioeconomic) and with a p-value $<0.10$ within the Wald test. The conclusion was obtained. Once the binary logistic regression analysis was carried out with a significance level of $p<0.10$, it can be identified that the variables WORKS and TYPE OF HOUSING meet the aforementioned
\end{abstract}

Información del manuscrito:

Fecha de recepción: 17 de enero de 2022.

Fecha de aceptación: 25 de febrero de 2022.

Fecha de publicación: 03 de marzo de 2022. 
criteria and are variables of significance in the problem of desertion of university students of the Faculty of Informatics Sciences of the Technical University of Manabí.

Keywords: Repetition, Multiple correspondence analysis, University, sociodemographic variables.

\section{Introducción}

Según Muñoz y Olivera (2015) a través de la historia el concepto de universidad ha evolucionado de tal forma que hoy día no sólo es concebida como una institución en donde es impartida la enseñanza superior mediante escenarios de discusión y difusión del saber, sino que es también un espacio en el que se promueve el desarrollo de competencias (lógicas, interpretativas, argumentativas, propositivas) para la adopción de roles y actitudes reflexivas, críticas y creativas que permitan la resolución de problemáticas en el contexto social.

De ahí que resulte importante que la educación que en ésta se imparte se encuentren a la vanguardia de la dinámica evolutiva de la sociedad, en vista de que en dicha sociedad es en donde los sujetos aplicaran sus conocimientos para el mejoramiento de su entorno.
La educación superior ha experimentado cambios significativos que permiten establecer su regulación, que en conjunto con el fortalecimiento económico y el crecimiento de la demanda en las universidades permiten brindar la formación técnica de tercer nivel de excelencia académica. (Falcón \& Jimenez, 2015)

Así mismo la Educación Superior se convierte en un factor social $y$ cooperativa que contribuye con el desarrollo de país, y por lo tanto su acceso debe ser de carácter gratuito, promoviendo la generación de profesionales idóneos con principios y normas basados en la igualdad de oportunidades. (Ajitimbay, Procel, \& Silva, 2019) La Educación Superior, sin perjuicio de los fines específicos de cada campo del saber, despertará en los educandos un espíritu reflexivo, orientado al logro de la autonomía personal, en un marco de libertad de pensamiento y de 
pluralismo ideológico que tenga en cuenta la universalidad de los saberes y la particularidad de las formas culturales existentes en el país.

El Ecuador según el Senescyt (Secretaría de la Educación y el Conocimiento) tiene una extensa ofertan académica en la educación superior, para responder a las necesidades de incrementar la competitividad a nivel nacional por medio de la innovación. De tal forma que nivel nacional existe cerca de 50 instituciones de educación superior (IES) acreditadas para el desarrollo de actividades académicas que acoge a cerca de 100000 bachilleres que culminan sus estudios secundarios y que postulan para conseguir un cupo en las mencionadas IES.

Las Instituciones de Educación Superior en su afán por formar sujetos integrales cada vez más, apuntan a mejorar sus planes curriculares y de acción, como un intento por responder a las demandas de los supuestos y necesidades que emergen en la sociedad, adoptando metodologías que les permitan avanzar $y$ evolucionar, buscando constantemente el desarrollo de competencias en los sujetos, para formarlos como seres capaces de transformar su entorno.

Por tal motivo, para lograr este objetivo es indispensable que los estudiantes se sientan comprometidos y responsabilizados por su proceso de formación, además una disposición constante que implique cambios en su conducta, esto es, que se mantengan activos, motivados $\mathrm{e}$ interesados durante su proceso de aprendizaje.

Sin embargo, cuando los aprendices no poseen la disposición y el compromiso con su formación tienden a presentarse ciertos obstáculos que impiden que el objetivo de formación profesional llegue a cumplirse. Entre estos obstáculos cabe mencionar el que concierne a la Repitencia, debido a que constituye uno de los problemas principales en el campo educacional. Cuando se presenta el caso de estudiantes repitentes que no han alcanzado los objetivos propuestos por la institución, se observa un déficit en torno a la formación de tales aprendices y por ende en la 
metodología y modelo pedagógico implementado por las IES.

En los últimos años las IES en Ecuador se observa un aumento en la reprobación de las asignaturas y por lo tanto los estudiantes se ven en la necesitad de recibir la catedra nuevamente. Esto puede ocurrir en consecuencia de varios factores que van a influir de una u otra manera en el rendimiento de los estudiantes a medida que cursan sus estudios universitarios (Passailaigue, Amechazurra, \& Galarza, 2014). Además, en el mes de diciembre correspondiente al año 2015 un $4.26 \%$ de jóvenes con edades entre 13 y 18 años que han cursado el mismo nivel académico más de una vez en lo que corresponde a los estudios superiores (Perez, Mendoza, \& Suarez, 2017).

La repitencia es un fenómeno que tiene incidencia en varios aspectos. En la parte personal afecta de forma emocional lo que puede conllevar a estados de depresión. En lo referente a lo institucional afecta la excelencia académica de forma negativa, además que el número de estudiantes aumenta significativamente. En la parte social aumenta las brechas de inequidad y desbalances sociales y en lo económico el gasto que conlleva es grande (Vargas, Bustos, \& Moreno, 2005). Además, se puede enmarcar que la repitencia se ha convertido en un problema de atención inmediata, que refleja el deficiente sistema de educación superior, que son la expresión de una pésima educación secundaria, así como también el desbalance entre la universidad y el mundo laboral (Bravo, Peña, \& Illesca, 2020)

Según Loyola y otros (2018) la repitencia estudiantil se define cuando el estudiante no consigue completar las competencias, además la considera como un indicador que establece la calidad académica deficiente que hay en una institución de educación superior. También se puede definir a la repitencia como la acción de repetir continuamente una actividad docente, debido a un rendimiento deficiente por parte del estudiante 0 por otro tipo de motivos ajenas a la parte académica.

Se puede identificar que la repitencia contribuyen al aumento de tasa de deserción, la baja retención en los estudiantes y un reducido número de estudiantes que pueden llegar a 
graduarse y que se representa en aspectos académicos dentro de las instituciones de educación superior (Martínez \& Torres, 2010).Hay que recalcar que el hecho de que los estudiantes pierdan una o varias asignaturas a lo largo de su formación universitaria, esto no indica que sea por la falta de capacidad del estudiante.

En relación a lo planteado, la repitencia representa una problemática y una deficiencia que afecta tanto a los estudiantes, y docentes como a la institución misma, en la medida en que se evidencia la ineficiencia en los procesos de formación, la falta de conocimiento alrededor del origen causal del problema y por consiguiente los escases de propuestas o alternativas de solución entorno a ésta.

El presente estudio se enfoca en determinar aquellos factores que influyen en la repitencia estudiantil mediante el análisis de correspondencia múltiple en la Facultad de ciencias Informáticas de la Universidad Técnica de Manabí. De igual manera se establece la siguiente pregunta de investigación: ¿Cuáles son los factores de mayor relevancia que influyen en la repitencia estudiantil de acuerdo al análisis de correspondencia múltiple en la Facultad de ciencias Informáticas de la Universidad Técnica de Manabí?

\section{Regresión logística}

Según Camarero y Albazán (2016) la regresión logística se define como la herramienta multivariante que tiene su aplicación en estudios tanto explicativo como predictivos y entre sus ventajas están el análisis de la información en investigación de cualquier área de conocimiento.

Además, la regresión logística permite realizar una clasificación fundamentadas en las características de la información, y como característica adicional los datos no necesitan tener la normalidad estricta que en conjunto con revisiones bibliográficas realizadas la definen con un instrumento indispensable para la categorización de la información.

\section{Regresión Logística Binaria}

Según establece AAA, se regresión logística binaria es factible cuando hay una variable dependiente dicotómica y un grupo de variables 
independiente ya sean cuantitativas o categóricas con la finalidad de realizar una estimación ajustada de la privacidad de que ocurra de un evento a partir de una o más variables independiente.

El modelo de regresión logística que representa la probabilidad de que un evento en mención pueda ocurrir se expresa de la siguiente manera:

$p=\frac{1}{1+e^{-\left(\beta_{0}+\beta_{1} X_{1}+\cdots+\beta_{k} X_{k}\right)}}$

En que $p$ representa la probabilidad de ocurrencia del evento 0 desenlace dicotómico estudiado, $Y$ es la variable dependiente (desenlace), $\mathrm{X}_{1}, \mathrm{X}_{2}, \ldots, \mathrm{X}_{\mathrm{k} s o n}$ las covariables y $\beta_{1}, \beta_{2} \ldots, \beta_{k}$ son los coeficientes de regresión asociados a cada covariable

\section{Materiales y métodos}

Se realiza una revisión literaria de artículos científicos en revista de alto impacto en temas referente a la aplicación de la regresión logística en una primera instancia para la recolección de información. A continuación, se realizará una revisión bibliográfica más exhaustiva limitando a artículos científicos que hayan surgido dentro los años más recientes.

Se tomarán en cuenta trabajos de investigación referentes a las instituciones de educación de superior, para identificar el uso de las técnicas multivariantes aplicadas a la repitencia estudiantil dentro de las instituciones de educación superior.

Se estableció una población de 200 estudiantes durante 5 periodos académicos (de 2017-S2 a 2019-2) que pertenecen a la Facultad de Ciencias informáticas de la Universidad Técnica de Manabí. Esta información fue extraída de la Dirección de las Tecnologías de Información de la UTM que genera el Sistema de Gestión Académica (SGA)

Para objeto del estudio se indicó la variable de Nivel de Repitencia Estudiantil como dependiente y dicotómica como se describe en la Tabla 1. 
Tabla 1. Equivalencias de repitencia.

\begin{tabular}{|l|l|}
\hline Porcentaje & Observación \\
\hline $0-50 \%$ & Repitencia Baja \\
\hline $51-100 \%$ & Repitencia Alta \\
\hline
\end{tabular}

Las variables independientes que han sido utilizadas son las que indican en la Tabla 2:

Tabla 2. Variable objeto del estudio

\begin{tabular}{|c|c|}
\hline Variable & Descripción \\
\hline Tipo_nacionalidad & $\begin{array}{l}\text { Tipo de nacionalidad del estudiante (ECUATORIANA, } \\
\text { CUBANA, COLOMBIANA Y DOMINICANA) }\end{array}$ \\
\hline Genero & $\begin{array}{l}\text { Tipo Biológico de genero del estudiante (Masculino y } \\
\text { Femenino) }\end{array}$ \\
\hline Estado_civil & $\begin{array}{l}\text { Estado civil del estudiante, se divide en las siguientes } \\
\text { categorías: Soltero, Casado y Unión Libre }\end{array}$ \\
\hline Sostenibilidad & $\begin{array}{l}\text { Especifica el tipo de plantel del cual proviene el estudiante } \\
\text { universitario. Dentro de Ecuador los planteles educativos se } \\
\text { dividen en las categorías: Fiscal, fiscomisional, municipal y } \\
\text { particular }\end{array}$ \\
\hline Internet_dispone & Acceso a redes de internet en su lugar de residencia. \\
\hline Tipo_vivienda & $\begin{array}{l}\text { Indica el tipo de vivienda en donde el estudiante vive, se divide } \\
\text { entre varias categorías: Apartamento/Casa, Bloque, Casa } \\
\text { Popular y Rancho. }\end{array}$ \\
\hline Tipo_salario_ingreso_total & $\begin{array}{l}\text { Especifica el valor total del ingreso familiar donde el estudiante } \\
\text { vive. Existen varias categorías: } \\
>1095 \text { a } 1444 \text { dólares, } \\
>1445 \text { a } 3494 \text { dólares, } \\
>945 \text { a } 1094 \text { dólares, } \\
>770 \text { a } 944 \text { dólares, } \\
>601 \text { a } 769 \text { dólares, } \\
>500 \text { a } 600 \text { dólares, } \\
>\text { menos de } 499 \text { dólares. }\end{array}$ \\
\hline Vivienda_localización & $\begin{array}{l}\text { Indica el lugar donde se encuentra ubicada la vivienda del } \\
\text { estudiante, existen varias categorías: Barrio, Cerro o Semi } \\
\text { Rural, Sector Popular, Urbanización. }\end{array}$ \\
\hline $\begin{array}{l}\text { Nivel de educación del jefe } \\
\text { familiar }\end{array}$ & $\begin{array}{l}\text { Indica que nivel de instrucción académico alcanzo el jefe } \\
\text { familiar, entre las categorías tenemos: } \\
\text { NO PROFESIONAL, TECNICO, UNIVERSITARIO. }\end{array}$ \\
\hline
\end{tabular}

Para el análisis de los datos se hizo uso del software estadístico SPSS, por pasos hacia atrás haciendo uso del criterio de Razón de Verosimilitud (RV) entre las variables dependiente
(Repitencia Estudiantil) y las variables independientes (Socioeconómicas) y con un $\mathrm{p}$ valor $<0.10$ dentro del test de Wald. 
Tabla 3. Test de Wald

\begin{tabular}{|c|c|c|c|c|c|c|c|}
\hline & & B & $\begin{array}{l}\text { Error } \\
\text { estándar }\end{array}$ & Wald & gl & Sig. & $\operatorname{Exp}(B)$ \\
\hline \multirow[t]{12}{*}{ Paso $1^{\text {a }}$} & tipo_nivel_ingreso2 &,- 069 & .056 & 1,532 & 1 & ,216 & ,933 \\
\hline & $\begin{array}{l}\text { nivel_estudio_jefe_hogar } \\
2\end{array}$ & ,088 &, 125 & .489 & 1 & ,485 & 1,092 \\
\hline & TRABAJA & .446 & ,211 & 4,463 & 1 &, 035 & 1,562 \\
\hline & DISPONEINTERNET2 &,- 082 &, 265 & .097 & 1 &, 756 & ,921 \\
\hline & ESTADOCIVIL2 &,- 551 & .470 & 1,375 & 1 & 241 &, 576 \\
\hline & SOSTENIBILIDAD2 &, 023 &, 076 & .089 & 1 &, 766 & 1,023 \\
\hline & TIPOVIVIENDA2 &, 244 &, 122 & 4,000 & 1 & .045 & 1,277 \\
\hline & SECTORRESIDENCIA2 &, 151 &, 116 & 1,711 & 1 & 191 & 1,163 \\
\hline & $\begin{array}{l}\text { PARROQUIAPROCEDEN } \\
\text { CIA2 }\end{array}$ &,- 416 & ,260 & 2,552 & 1 & 110 &, 660 \\
\hline & EDAD &, 060 &, 037 & 2,652 & 1 & ,103 & 1,062 \\
\hline & GENERO2 &,- 017 &, 238 &, 005 & 1 & 941 &, 983 \\
\hline & Constante & 582 & 1,426 & 167 & 1 & 683 & 1,790 \\
\hline
\end{tabular}

\section{Resultados y discusión}

Se puede observar los siguientes resultados entre la variable dependiente y las variables socioeconómicas que se analizaron dentro del estudio:

La variable de tipo_nivel_ingreso tuvo un resultado de fue de 0.933 , pero se puede evidenciar que en la relación estadística no hubo significancia. Con respecto a la variable nivel_estudio_jefe_hogar se evidencio un valor del 1,092. La variable de trabaja presenta un valor de 1,092 y muestra un nivel de significancia el 0,035. La variable dispone internet presenta un valor de 0,921 con un nivel de significancia de 0.756 . Haciendo hincapié a la variable estado civil se puede observar que muestra un resultado
0.576 con un nivel de significancia del 0,241 La variable sostenibilidad nos indica un resultado del 1,023 con un nivel de significancia de 0,766. Al respecto de la variable tipo vivienda muestra un resultado de 1,277 y además presenta un nivel de significancia de 0,045 . La variable de sector residencia muestra un resultado de 1,163 y además indica un nivel de significancia del 0,191. También se puede observar que la variable parroquia residencia presenta un resultado de 0,660 con un valor de significancia de 0,110. La variable edad presenta un resultado de 1,062 con un nivel de significancia de 0,103 . Adicional se muestra que la variable genero establece un resultado de 0,983 en conjunto con un nivel de significancia de 0,941. 
Al decir de Almuiñas, Galarza, y otros (2016), la repitencia de los estudiantes del nivel universitario constituye un problema complejo en el cual inciden diversos factores que pueden agruparse en dos grandes campos que son:

a. Factores exógenos al proceso docente-educativo, como la condición socioeconómica, composición familiar, vivienda, nutrición, actitudes, valores familiares frente a la educación, nivel educacional de los padres, capacidades cognitivas, motivación por la carrera, nivel académico alcanzado por los estudiantes en la enseñanza media, etc.

b. Factores endógenos como políticas educacionales de las instituciones, recursos humanos, materiales y financieros de las IES, relaciones pedagógicas, normas organizativas, métodos, contenidos y orientación de la enseñanza, dificultad "objetiva" de la carrera, desempeño de los docentes, entre otros.

Los valores resultantes del proceso multivariado permiten establecer que la repitencia en estudiantes universitarios influye varios factores, uno de mayor relevancia que el otro, lo cual establece que la problemática es multicausal, debiendo ser motivo de análisis no solo de la sociedad sino también de las instituciones de educación superior.

De ahí que la repitencia constituya un problema de gran interés, en tanto que se ha convertido en una de las mayores dificultades que obstaculiza epistemológica, intelectual, personal y académicamente el desarrollo del aprendizaje optimo del estudiante, limitando su participación y activa colaboración durante su proceso de formación. (Martelo \& Acevedo, 2017)

\section{Conclusiones}

Una vez realizado el análisis de la regresión logística binaria con un nivel de significación de $p<0.10$ se puede identificar que las variables TRABAJA y TIPO DE VIVIENDA cumple con el criterio mencionado. Es decir, que estás variables son de significancia en la problemática de deserción de estudiantes universitarios de la Facultad de Ciencias Informáticas de la Universidad Técnica de Manabí. Le 
siguen en relevancia las variables el sector de residencia y sostenibilidad que hace referencia al colegio de donde provienen siendo las instituciones fiscales las que manifiestan dificultades en conocimientos para la carrera de informática.

Dentro de la evaluación en las IES aparece la problemática relacionada con la repitencia, como fenómeno que está presente. Esta problemática se encuentra influenciada por factores exógenos y endógenos y tienen implicaciones individuales, colectivas, institucionales y sociales. Este constituye un problema a resolver también en las IES ecuatorianas, las que deben enfocarse al incremento de estudios diagnósticos en este campo y en consecuencia, proponer acciones de cambio para minimizar el efecto de estos fenómenos en los propios estudiantes, las instituciones universitarias y la sociedad.

Entre los retos que la IES deben asumir se encuentran, por ejemplo: el perfeccionamiento del sistema de ingreso, la flexibilización de las normativas académicas; el completamiento de la base material de estudio; la superación continua de los profesores a tiempo completo y parcial; el perfeccionamiento de los planes de estudio que considere al estudiante como el centro del proceso docente educativo, por mencionar solo algunas de las estrategias más importantes.

\section{Bibliografía}

Ajitimbay, C., Procel, A., \& Silva, L. (2019). Factores que intervienen en la repitencia estudiantil. Ecuador: Universidad Ciencia y Tecnología.

Almuiñas, J., \& Galarza, J. (2016). Estudio sobre bajas y la repitencia en carreras e IES de Cuba. Obtenido de file://C:/Users/User/Downloa ds/177-

Texto\%20del\%20art\%C3\%A Dculo-177-1-1020161128.pdf

Bravo, F., Peña, M., \& Illesca, L. (2020). Reprobación y abandono de estudiantes universitarios. Obtenido de http://45.238.216.13/ojs/index .php/EPISTEME/article/view/ 1728

Camarero, L., \& Almazán, A. (2016). Regresión logística: fundamentos y aplicación a la investigación sociológica. Obtenido de https://www2.uned.es/socioes 
tadistica/Multivariante/Odd_R atio_LogitV2.pdf

Cerpa, W. (2015). Análisis multivariado para determinar los factores más relevantes de deserción estudiantil presentes en el programa de Ingeniería Industrial de una universidad colombiana. Colombia: Prospectiva.

Céspedes, J., \& Cardoso, G. (2010). Análisis de componentes principales y análisis de regresión de datos categóricos. México: Revista matemática: teoría y aplicaciones.

Falcón, D., \& Jiménez, A. (2015). Los indicadores actuales de la eficiencia académica: necesidad de su perfeccionamiento. México: Pedagogía universitaria.

García, M., \& Alvarado, J. (2016). La predicción del rendimiento académico: regresión lineal versus regresión logística. Obtenido de https://www.redalyc.org/articu lo.oa? id=72797059

Loyola, E. (2018). Repitencia estudiantil en la Universidad Politécnica Salesiana. Obtenido de https://rraae.cedia.edu.ec/Re cord/UPS_4abf4beff0251d8c e21860aab52a6cc7

Lozares, C., \& López, P. (2010). El análisis de componentes principales: aplicación al análisis de datos secundarios. México: Revista de sociología.

Mares, A. (2020). Optimización en manufactura mediante experimentación y modelos de regresión logística. Obtenido de http://portal.amelica.org/ameli /jatsRepo/339/3391369014/ht $\mathrm{ml} /$ index.html

Martelo, R., \& Acevedo, D. (2017). Análisis multivariado aplicado a determinar factores clave de la deserción universitaria. Obtenido de https://www.revistaespacios.c om/a18v39n10/a18v39n10p1 3.pdf

Martínez, V., \& Torres, L. (2010). Análisis de los hábitos de estudio en una muestra de alumnos universitarios. Obtenido de https://pdfs.semanticscholar.o rg/7943/e3649688a3a02356f 3c713cedd57de62ea9c.pdf

Muñoz, R., \& Olivera, M. (2015). Repitencia del programa de medicina en una institución de educación superior. Obtenido de https://biblioteca.utb.edu.co/n otas/tesis/0069848.pdf

Passailaigue, R., Amechazurra, O., \& Galarza, J. (2014). La deserción y la repitencia en las instituciones de educación superior:

algunas 
experiencias investigativas en

el Ecuador. Obtenido de file:///C:/Users/User/Downloa ds/177-

Texto\%20del\%20art\%C3\%A Dculo-177-1-1020161128.pdf

Pérez, C., Mendoza, E., \& Suarez, R. (2017). Deserción y repitencia en estudiantes de la carrera de Enfermería matriculados en el período 2010-2015. Universidad Técnica de Manabí. Obtenido de https://medes.com/publication $/ 143332$

Torrado, M., \& Berlanga, V. (2012). Análisis discriminante mediante SPSS.REIRE. México: Revista de innovación en educación.

Vargas, J., Bustos, L., \& Moreno, R. (2005). Propuesta para aumentar el nivel académico, minimizar la deserción, rezago y repitencia universitaria por problemas de bajo rendimiento académico en la Universidad tecnológica de Pereira, en el programa ingeniería de sistemas y computación. Obtenido de https://revistas.utp.edu.co/ind ex.php/revistaciencia/article/v iew/6841 